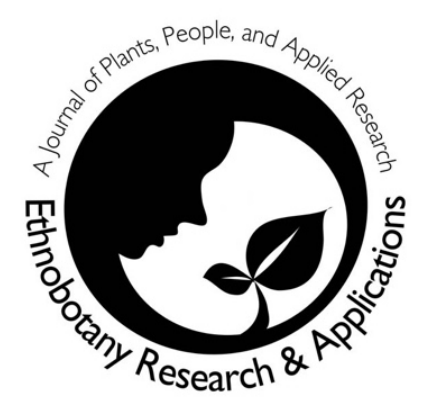

\title{
Agracejo: Muchas especies, escasa información etnobotánica y etnofarmacológica
}

\author{
Cristhian N. Rodríguez-Silva, J-Kenedy Ramirez, Sharon \\ Velasquez-Arevalo, Víctor E. Villarreal-La Torre.
}

\section{Mini Review}

\begin{abstract}
Resumen
Antecedentes: La diversidad botánica del Perú ha hecho que actualmente se esté promoviendo el uso de plantas medicinales en las instituciones de salud a nivel nacional. El objetivo de esta revisión es proporcionar información científica que respalde las propiedades terapéuticas del agracejo como Berberis vulgaris.
\end{abstract}

Métodos: Se buscó información en bases de datos científicas, Plantlist, Scopus, PubMed, Google Académico y repositorios de tesis universitarias sobre, información etnobotánica y etnofarmacológica y se comparó con el Berberis rigida Hieron, Berberis vulgaris, Ilex guayusa Loes, Vellesia glabra (Cav), dado que todas ellas presentan también el nombre común de agracejo.

Resultados: Actualmente existen 3 familia y 4 géneros que presentan a una especie conocida como agracejo, se destaca 5 tesis universitarias peruanas en los últimos 10 años que abordan las utilidades del género Berberidaceae y sus especies, teniendo como mayor utilidad la tintura. La mayor información científica, 1363 estudios entre etnobotánico y etnofarmacológicos, respaldan la utilidad del $B$. vulgaris, aunque son provenientes de países orientales y solo 3 son peruanas.

Conclusiones: La información que respalda la utilidad del B. vulgaris (agracejo), proviene de publicaciones internacionales (Irán, Pakistán), además no se cuenta con información científica etnobotánica y etnofarmacológica peruana, esto genera un riesgo de confusión de especies, más aún si presenta el mismo nombre común, y características fitoterapéuticas similares. Estas dos situaciones originarían que se adopte información de otros países, lo que a su vez pone en riesgo de posible inefectividad del tratamiento con medicina tradicional.

Palabras claves: Berberis, indicación fitoterapéutica, medicina tradicional, plantas medicinales, agracejo, metabolitos secundarios, berberidaceae, aquifoliaceae, apocynaceae.

\section{Correspondence}

Cristhian N. Rodríguez-Silva*, J-Kenedy Ramirez, Sharon Velasquez-Arevalo, Víctor E. Villarreal-La Torre

Facultad Farmacia y Bioquímica, Universidad Nacional de Trujillo. Av. Juan Pablo II s/n. 13011. Trujillo-Perú

*Corresponding Author: crodriguezsi@unitru.edu.pe

\section{Ethnobotany Research \& Applications}

19:17 (2020)

\section{Antecedentes}

La sociedad actual, en su búsqueda de tratar de disminuir sus problemas de salud, recurre a diversos mecanismos terapéuticos, muchos de estos no son convencionales, a los que se les conoce como medicina complementaria (MC) medicina alternativa (MA), medicina natural (MN) o simplemente medicina tradicional (MT) (OMS 2010). El National Center for Complementary and Alternative (NCCAM) de la National Institutes of Health (NIH), afirma que la medicina complementaria y alternativa (MCA), es una sistematización de procesos de asistencia sanitaria que de forma paralela al tratamiento médico convencional se complementan para 
contribuir a alcanzar el objetivo terapéutico. (NCCAM 2015).

La biodiversidad fitológica peruana y sus datos históricos de la utilidad terapéutica de plantas utilizadas por su efecto medicinal, ha incrementado el interés de muchas empresas a conocer, procesar y/o comercializar especies fitoterapéuticas como productos herbarios (OPS 2018). Existen diversas instituciones nacionales e internacionales dedicadas a la investigación de la etnomedicina y etnobotánica, sin embargo, aún no se cuenta con un registro único y estandarizado de las características de las plantas estudiadas, es decir, los conocimientos y técnicas tradicionales se encuentran dispersos y $\sin$ sistematizar, lo que puede provocar diversos inconvenientes como la confusión del nombre científico de una especie con otra (sinonimia) o que especies de género distintos posean el mismo nombre común (homonimia). Estos inconvenientes se podrían evitar si existiera información adecuada y completa de la especie, por ello es fundamental la certificación botánica desde su registro taxonómico hasta su identificación, cuantificación y caracterización de metabolitos secundarios, así como estudios in vitro e in vivo, como paso previo para su correcto uso medicinal (OPS 2018, Marinoff et al 2009). Las tendencias actuales, señalan que se ha incrementado la disposición de pacientes para recibir tratamiento natural, ya sea para uso preventivo o como coadyuvante al tratamiento farmacológico, resaltado en los 90000 pacientes atendidos en medicina complementaria de EsSaludPerú (OPS 2018). Las Farmacias Naturales en los Centros de Atención de Medicina Complementaria de EsSalud, dispensan las hojas y tallos del Berberis vulgaris (agracejo) como depurativo y desintoxicante natural, aunque también se le reconoce otros efectos tales como diurético y antihipertensivo (EsSalud 2016), no obstante, existen datos etnobotánicos que este nombre común se refiere también a otras tres especies de familias distintas Berberidáceae, Aquifoliaceae y Apocynaceae Tabla 1)

Tabla 1. Clasificación taxonómica de las diferentes especies de agracejo

\begin{tabular}{lllll}
\hline Familia & Berberidaceae & Berberidaceae & Aquifoliaceae & Apocyneaceae \\
\hline Género & Berberis & Berberis & Ilex & Vellesia \\
\hline Especie & $\begin{array}{l}\text { Berberis rigida } \\
\text { Hieron }\end{array}$ & $\begin{array}{l}\text { Berberis vulgaris } \\
\text { L }\end{array}$ & $\begin{array}{l}\text { llex guayusa } \\
\text { Loes }\end{array}$ & $\begin{array}{l}\text { Vallesia glabra (Ruiz } \\
\text { \& Pav.) }\end{array}$ \\
\hline
\end{tabular}

El objetivo de esta revisión es proporcionar información científica que respalde las propiedades terapéuticas del Berberis vulgaris (agracejo), así mismo comparar las características etnobotánicas, fitoconstituyentes y propiedades terapéuticas con 3 especies homónimas.

\section{Materiales y Métodos}

Esta revisión fue elaborada a partir de la búsqueda en bases de datos de Plantlist, Scopus, PubMed y Google Académico (como motor de búsqueda) para compilar investigaciones sobre el tema de investigación en actividad fitoterapéutica de las especies peruanas de agracejo, además se complementó las búsquedas en base de datos locales: Alicia, Renati y repositorios de tesis universitarias.

\section{Resultados y discusión \\ Familia Berberidaceae}

La familia Berberidaceae, es nativa de países ubicados en el centro y sur de Europa, Noreste africano y Noroeste asiático principalmente en los países de Irán y Pakistán (Minaiayan Et al. 2011). En Latinoamérica, su distribución geográfica empieza en México y llega hasta la Patagonia en Chile. Esta familia consta de 14 géneros y 701 especies en el mundo (Vásquez \& Rojas 2016), entre ellos el género Berberis, con 650 especies registradas en el mundo (Ulloa 2006), así mismo, en América del Sur se encuentran 99 especies distribuidas entre Bolivia (15 especies), Argentina (20 especies), Ecuador (32 especies) y Perú (32 especies) de los cuales 14 especies son endémicas y dos en áreas naturales protegidas por el Estado peruano (Ulloa 2006).

Las características botánicas de las Berberidaceaes son, en su mayoría arbustos de forma leñosa, puede presentar espinas; sus hojas alternas, radicales o fasciculadas sobre las ramitas atrofiadas (Braquielados), con o sin estípulas simples (en las especies peruanas) o compuestas, membranáceas hasta coriáceas; las flores son hermafroditas, cíclicas, actinoformas, generalmente amarillas, solitarias o en racimos, espigas o panículas. El fruto es una baya, cápsula o raramente aquenio. Semilla con endospermo abundante, con o sin arilo. (Mostacero et al. 2002).

La finalidad de los estudios etnobotánicos es aportar con la sistematización del uso tradicional de las plantas para que sirva de base para investigaciones farmacológicos in vitro e in vivo y tecnológicos, es decir darle forma farmacéutica adecuada para el o 
los metabolito activo aislados; cabe resaltar que estos estudios y sus publicaciones pueden ayudar a mejorar la salud de la población y disminuir el gasto en atención primaria en el sistemas de salud (Bussmann 2015). En los últimos 10 años solo se ha encontrado 5 estudios nacionales que aporten datos etnobotánicos de familia de Berberidaceas (Tabla 2), allí se informa de los géneros y especies que se encuentran en diversas ciudades en diferentes alturas, no obstante, en todas ellas se afirman que el uso principal del género berberis es el de tintura (preparado donde la extracción de principios activos se hace macerando la droga vegetal y una mezcla de solvente agua-alcohol), especialmente el fruto y parte de la corteza.

Tabla 2. Estudios en los últimos años que aportan conocimiento general sobre las Berberidaceaes en regiones altoandinas del Perú

\begin{tabular}{|c|c|c|c|c|c|}
\hline Ciudad & $\begin{array}{l}\text { Altura } \\
\text { (m) }\end{array}$ & Título & Especie encontrada & Uso tradicional & Referencia \\
\hline Junín & 3050 & $\begin{array}{l}\text { Caracterización de los } \\
\text { arbustos en la provincia de } \\
\text { Tarma, departamento de } \\
\text { Junín, con énfasis en su } \\
\text { morfología vegetativa }\end{array}$ & $\begin{array}{l}\text { Berberis lutea Ruiz \& } \\
\text { Pav }\end{array}$ & $\begin{array}{l}\text { Colorante } \\
\text { (Amarillo)de } \\
\text { vestimenta. } \\
\text { Fruto maduro } \\
\text { color de bebidas }\end{array}$ & $\begin{array}{l}\text { Saavedra S. } \\
2017\end{array}$ \\
\hline Cajamarca & 3000 & $\begin{array}{l}\text { Etnobotánica y } \\
\text { características } \\
\text { morfológicas de la } \\
\text { vegetación leñosa en un } \\
\text { remanente de bosque de } \\
\text { la microcuenca Río } \\
\text { Grande, la encañada - } \\
\text { Cajamarca }\end{array}$ & $\begin{array}{l}\text { Berberis jelskiana } \\
\text { C.K.Schneid. }\end{array}$ & $\begin{array}{l}\text { Fruto como } \\
\text { alimento y la } \\
\text { corteza como } \\
\text { colorante de } \\
\text { vestimenta. }\end{array}$ & $\begin{array}{l}\text { Alva E. } \\
2017\end{array}$ \\
\hline Cajamarca & 2252 & $\begin{array}{l}\text { Etnobotánica de la flora } \\
\text { arbórea y arbustiva del } \\
\text { Departamento de } \\
\text { Cajamarca }\end{array}$ & $\begin{array}{l}\text { Berberis jelskiana } \\
\text { C.K.Schneid. } \\
\text { Berberis lutea Ruiz \& } \\
\text { Pav. }\end{array}$ & $\begin{array}{l}\text { La corteza, el } \\
\text { fruto y ramas } \\
\text { usos como } \\
\text { colorantes }\end{array}$ & $\begin{array}{l}\text { Llanos J. } \\
2018\end{array}$ \\
\hline Ayacucho & 2900 & $\begin{array}{l}\text { Flora arbórea y arbustiva } \\
\text { del bosque de Ustuna, } \\
\text { centro poblado Santa } \\
\text { Isabel de Chumbes, } \\
\text { distrito Ocros, provincia } \\
\text { Huamanga. Ayacucho, } \\
2016 .\end{array}$ & $\begin{array}{l}\text { Berberis lutea Ruiz \& } \\
\text { Pav } \\
\text { Berberis sp. }\end{array}$ & $\begin{array}{l}\text { El fruto utilizado } \\
\text { como colorante } \\
\text { de lana y } \\
\text { vestimenta }\end{array}$ & $\begin{array}{l}\text { Carpio J. } \\
2017\end{array}$ \\
\hline Cusco & 2687 & $\begin{array}{l}\text { Etnobotánica y fitoquímica } \\
\text { de plantas tintóreas en las } \\
\text { comunidades de Rumira, } \\
\text { Chaullacocha y Chupani: } \\
\text { provincia de Urubamba- } \\
\text { Cusco }\end{array}$ & $\begin{array}{l}\text { Berberís lutea Lechler } \\
\text { Berberís lutea var. } \\
\text { conforta (Kunth) DC } \\
\text { Berberís carinata } \\
\text { Lechler }\end{array}$ & $\begin{array}{l}\text { Colorante } \\
\text { amarillo de lana } \\
\text { y vestimenta }\end{array}$ & $\begin{array}{l}\text { Gutiérrez \& } \\
\text { Puelles. } \\
2012\end{array}$ \\
\hline
\end{tabular}

Fuente: Alicia Concytec 2020.

\section{Género Berberis}

\section{Distribución}

Este género se puede encontrar en todos los continentes, aunque predominante en países orientales como, Irán, Pakistán, Japón y China (Khan et al. 2016), también se puede encontrar en algunos países de América del Sur, y se encuentra en zonas con climas templados. En Perú se recolectan de las regiones mesoandinas, que comprende desde la región ecológica Yunga a Suni, entre los 1200 y 4200 m de altura. En la región La Libertad se encuentran tres especies $B$. buceronis G.F. Macbride, B. loxensis Bentham, B. weberbaueri (Aredo et al. 2017), siendo la B. buceronis una de las especies endémicas circunscritas además a los departamentos Piura, Lambayeque, Cajamarca y San Martin (Galán de Mera et al. 2017).
El género Berberis, es un arbusto espinoso y se caracteriza por coloración amarilla de la hojas y flores, el fruto puede presentar diversos colores, entre el azul y rojo, dependiendo de la especie y el nivel de maduración (Salehi et al. 2019).

\section{Fitoquímica del género Berberis}

El género Berberis ha evidenciado estudios con actividad antibacteriana y beneficios hepáticos (Bussmann et al. 2009a, Bussmann et al. 2010), pues presenta diversos fitoconstituyentes como flavonoides, saponinas, cardiotónicas y esteroides (Bussmann et al. 2009b), además alcaloides como principales metabolitos terapéuticos, entre ellos los derivados isoquinolínicos como la berberina (Fig. 1 A) y berbamina (Fig. 1B), quienes provienen del 
metabolismo de la tirosina (Srivastava S. 2015, Mokhber-Dezfuli et al. 2014). En la Figura 2, se observa el esquema general de metabolitos presentes en las especies del género Berberis.<smiles>COc1ccc2cc3[n+](cc2c1OC)CCc1cc2c(cc1-3)OCO2</smiles>

(A) Berberina<smiles>COc1cc2c(cc1OC)C(Cc1ccc(Oc3ccc(O)c(Oc4ccc5c(c4OC)C(Cc4cccc(O)c4)N(C)CC5)c3)cc1)N(C)CC2</smiles>

(B) Berbamina

Fig. 1. Principales alcaloides del género Berberis.
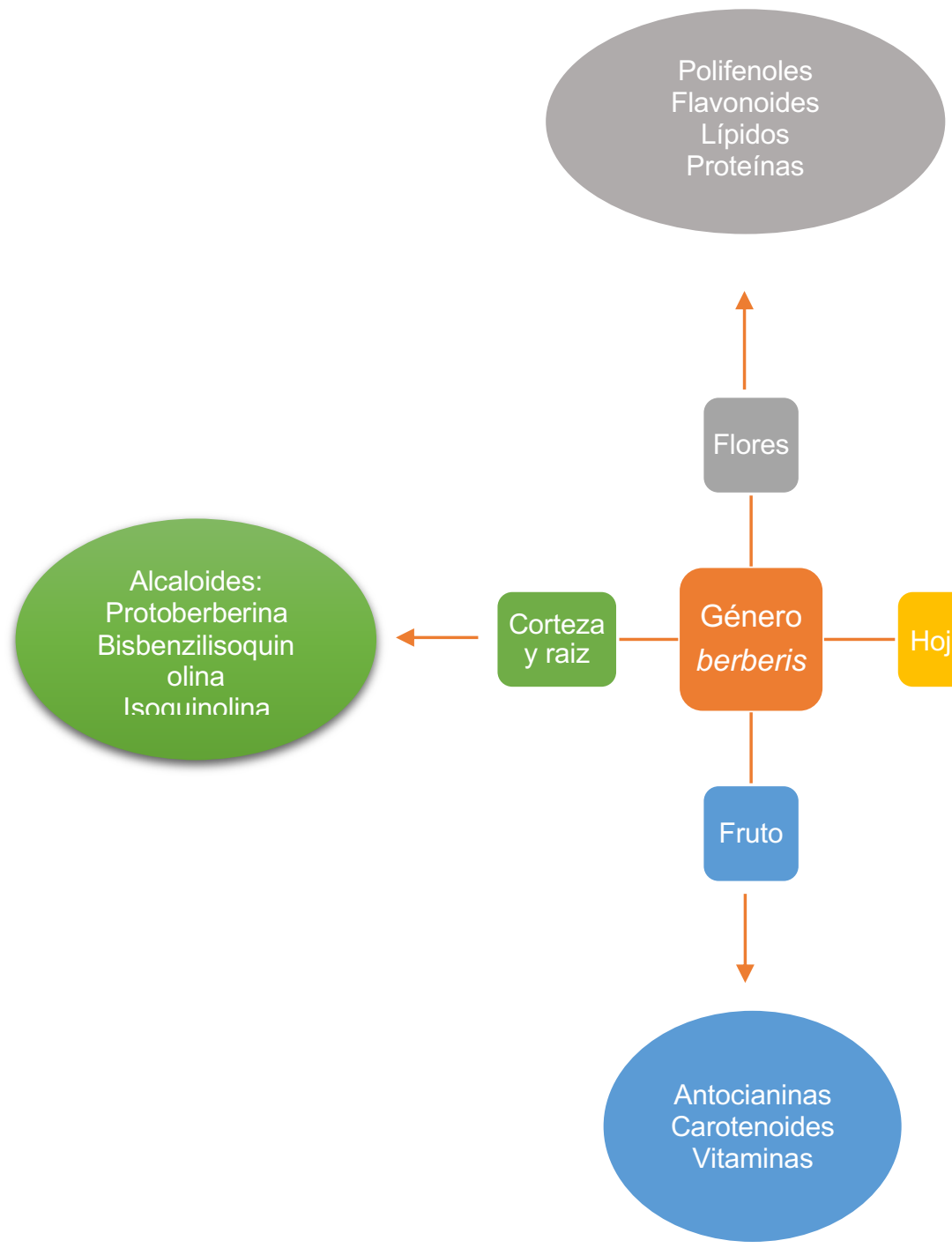

Figura 2. Esquema de metabolitos presentes en el género Berberis

Berberis vulgaris L., es la especie más conocida de este género y distribuida en varios países de todos los continentes en muchos de ellos toma diversos nombres (Tropicos.org 2020), en el Perú se le conoce, comercializa y dispensa como agracejo. Las informaciones sobre las características botánicas provenientes de fuentes internacionales y peruanas son similares en las diversas partes de la planta. Es un arbusto que mide entre 1 a $3 \mathrm{~m}$ de altura, espinoso con madera de color amarillo, hojas coriáceas ovaladas, flores en racimo de color amarillo, el fruto es una baya de forma oblonga. La 
raíz, corteza y fruto se utilizan con fines terapéuticos en afecciones hepáticas, intestinales y cálculos renales (Javadzadeh \& Fallah 2012, Roersch 1994,
Salehi et al. 2019, Mostacero et al. 2002) (Tabla 3), las estructuras de sus principales metabolitos se muestran en la Figura 3.

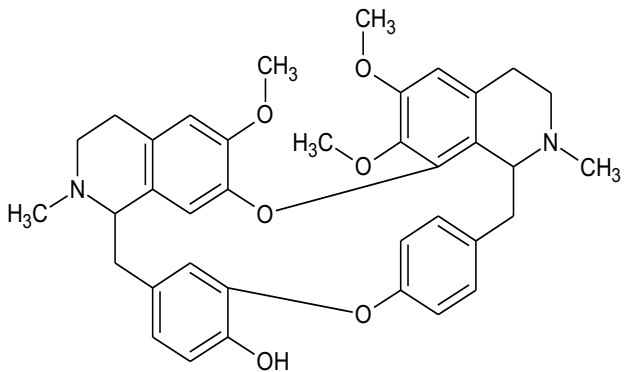

Oxicantina<smiles>COc1cc2c(cc1OC)-c1cc3ccc(OC)c(OC)c3c[n+]1CC2</smiles>

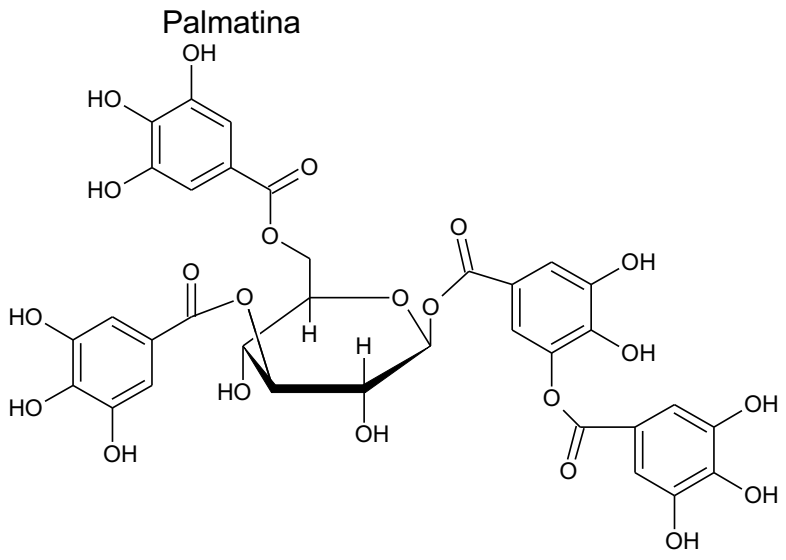

Taninos<smiles>CC1(C)C(O)CC[C@]2(C)C1CC[C@]1(C)C3CCC4(C(=O)O)CCC5(C)CC5(C)CC4C3CCC21</smiles><smiles>O=C(O)CC(O)C(=O)O</smiles>

ácido oleanólico

ácido málico

Columbamina<smiles>COC1=C(OC)C2=C(CC1)CC1c3cc4c(cc3CCN1C2=O)[O+](C)CO4</smiles><smiles>O=C1O[C@H]([C@H](O)CO)[C@H](O)C1O</smiles>

ácido ascórbico

Las hojas de $B$. vulgaris, también presenta aceites esenciales como<smiles>Cc1ccc(C(C)C)cc1</smiles>

p-cymeno<smiles>C=C(C)[C@H]1CC=C(C)CC1</smiles>

limoneno<smiles>C=C/C(C)=C/CCC(=C)C</smiles>

ocimeno.

Figura 2. Estructuras de metabolitos más importantes presentes en Berberis vulgaris. 
Tabla 3. Efectos farmacológicos comunes y fitoconstituyentes del Berberis vulgaris

\begin{tabular}{|c|c|c|c|c|}
\hline Parte & Efecto & $\begin{array}{c}\text { Forma de } \\
\text { uso }\end{array}$ & Fitoconstituyentes & Referencias \\
\hline Fruto & $\begin{array}{l}\text { Analgésico estomacal, } \\
\text { biliar y en las } \\
\text { articulaciones, antipirético, } \\
\text { efectivo para ciertas } \\
\text { infecciones como } \\
\text { garganta, uretra, dérmicas } \\
\text { (Acné Vulgar) } \\
\text { gastrointestinal, pulmonar. } \\
\text { Dismenorrea, Purificador } \\
\text { de la sangre (depurativo), } \\
\text { antiemético, } \\
\text { antihipertensivo } \\
\text { (Diurético), antiarrítmico } \\
\text { (inotrópico positivo*) } \\
\text { hipocolesterolémico. } \\
\text { Nutriente alimenticio+. } \\
\text { Hipoglucemiante. }\end{array}$ & $\begin{array}{l}100 \mathrm{~g} \text { de } \\
\text { fruto } \\
\text { decocto } \ddagger \text { en } \\
1 \text { litro de } \\
\text { agua }\end{array}$ & $\begin{array}{l}\text { Vitamina C, * Alcaloides } \\
\text { como berberina (1), } \\
\text { berbamina (2) } \\
\text { oxiacantina (3), } \\
\text { columbamina (4) y } \\
\text { berberubina (5). } \\
{ }^{+} \text {Contiene glucosa, } \\
\text { fructosa, ácido málico, } \\
\text { ácido oleanólico, ácido } \\
\text { tartárico, pectina y } \\
\text { resinas, Vitamina A, } \\
\text { Ca+2, Fe }{ }^{+3} \text { y K, } 2 \% \\
\text { proteína, } 16.24 \% \\
\text { carbohidratos y } 0.99 \% \\
\text { ceniza. La antocianina se } \\
\text { estima en } 281 \text { mg/L. }\end{array}$ & $\begin{array}{l}\text { Javadzadeh \& } \\
\text { Fallah } 2012 \\
\text { Seyyed Mahdi } \\
\text { Javadzadeh } \\
2013 \\
\text { Rahimi-Madiseh } \\
\text { et al. } 2017 \\
\text { Kalmarzi et al. } \\
2019 \\
\text { Kooti et al.2019 } \\
\text { Mostacero et al., } \\
2002\end{array}$ \\
\hline Hojas & $\begin{array}{l}\text { Dolor intestinal causado } \\
\text { por diarreas crónicas, } \\
\text { Colerético, colagogo. } \\
\text { Antihipertensivo, }\end{array}$ & $\begin{array}{l}15 \text { a } 30 \mathrm{~g} \text { de } \\
\text { hoja seca } \\
\text { decocto } \ddagger \text { en } \\
1 \text { litro de } \\
\text { agua. }\end{array}$ & $\begin{array}{l}\text { Vitamina C, Aceites } \\
\text { esenciales p-cymeno, } \\
\text { limoneno y ocimeno. }\end{array}$ & $\begin{array}{l}\text { Javadzadeh \& } \\
\text { Fallah } 2012 \\
\text { Salehi et al. } \\
2019\end{array}$ \\
\hline $\begin{array}{l}\text { Corteza } \\
\text { del tallo } \\
\text { y la raíz }\end{array}$ & $\begin{array}{l}\text { La infusión de la corteza } \\
\text { para el dolor hepático, } \\
\text { laxante, dolor artrítico, } \\
\text { antiinflamatorio } \\
\text { hemorroidal, etc. El } \\
\text { extracto acuoso de la raíz } \\
\text { se utiliza como antifúngico } \\
\text { y antileshmaniasico. } \\
\text { Diurético y Anticonceptivo. } \\
\text { Secretagogo, }\end{array}$ & $\begin{array}{l}20 \mathrm{~g} \text { de } \\
\text { corteza/tallo } \\
\text { o raíz } \\
\text { decocto }{ }^{\ddagger} \text { en } \\
1 \text { litro de } \\
\text { agua }\end{array}$ & $\begin{array}{l}\text { Raíz y corteza: dos } \\
\text { alcaloides fueron } \\
\text { identificados: } \\
\text { protoberberinas } \\
\text { (berberina, berbamina, } \\
\text { jateorrizine y palmatina) y } \\
\text { bisbencilisoquinolinas: } \\
\text { Oxicantina. } \\
\text { Otros alcaloides: } \\
\text { Verderina, palmatina, } \\
\text { columbamina, ácido } \\
\text { celedónico, vitamina C }\end{array}$ & $\begin{array}{l}\text { Javadzadeh \& } \\
\text { Fallah } 2012 \\
\text { Seyyed Mahdi } \\
\text { Javadzadeh } \\
2013 \\
\text { Mahmoudvand } \\
\text { et al. } 2014 \\
\text { Kooti et al.2019 } \\
\text { Mostacero et al., } \\
2002\end{array}$ \\
\hline
\end{tabular}

Esta especie se encuentra dentro del Petitorio Nacional de productos, recursos e insumos terapéuticos afines de uso en Medicina complementaria de EsSalud con el Código Sap 011250030 (EsSalud 2008) y su forma de dispensación en esta institución se da de acuerdo con la utilidad y necesidad de los pacientes e indicaciones, sus presentaciones son tintura $y$ extracto fluido (a partir de una tintura se concentra de los fitoconstituyentes por evaporación del solvente) (EsSalud 2016). El efecto antinflamatorio e inmunomodulador del extracto de Berberis vulgaris se debe a la berberina, probados en modelos 14 modelos in vivo y 10 modelos in vitro, y se da por alteración de la respuesta inmune celular de TH2 con inhibición de mediadores de la inflamación (TNF, IL-1 e IFN- Y), y aumento de la IL-4 e IL-10 (Kooti et al.2019).

El uso inadecuado e irregular del agracejo puede causar náuseas, regurgitación, vértigo, convulsiones, hemorragia nasal, insuficiencia renal, inflamación de la piel y los ojos y disminuir el azúcar en la sangre, además, no debe ser utilizada por niños, mujeres embarazadas y madres en periodo de lactancia (Javadzadeh \& Fallah 2012, Kalmarzi et al. 2019).

Berberis rigida Hieron, el portal Trópicos.Org, especializada en la consolidación de información y registros de las especies botánicas en el mundo, informa que el Perú no cuenta con registro, no obstante, si hay evidencia de esta especie en Ecuador, quien se nombra con el código (P.M. Jørgensen - 1207). Algunas características botánicas diferenciadas, es un arbusto espinoso que mide entre dos y tres metros de altura, con hojas amontonadas de $3 \mathrm{~cm}$ promedios, flores colgantes entre 5 a $8 \mathrm{~cm}$ de largo, frutos subglobosos carnosos de $8 \mathrm{~mm}$ de largo, que varían entre el verde al rojizo y finalmente negro morado. Sus frutos son comestibles. La madera sirve para construir cabos de herramientas. Antiguamente se usaba la madera amarilla para teñir fibras (Minga et al. 2016). Hay escasa información disponible que reportan aspectos etnomedicinales, pero se tiene 
conocimiento que los pobladores de Piura (Norte peruano) la conocen como agracejo.

\section{Otras especies conocidas como Agracejo} Ilex guayusa Loes (Aquifoliaceae): Agracejo en Perú, además, se le denomina como guayusa o citrodora (Bussmann \& Douglas 2015), se encuentra distribuida en regiones tropicales y subtropicales de Colombia, Brasil, Ecuador y Perú. Esta especie se ha encontrado entre los 0-1500 m altura (Radice \& Vidari 2007), aunque otros autores afirman que puede habitar entre 200 a 2600 m (Dueñas et al. 2016). Esta especie son árboles bastante altos y gruesos, alrededor de $10 \mathrm{~m}$ de altura, y $1 \mathrm{~m}$ de diámetro, sus hojas son coriáceas dentadas oblongas/elípticas de color verde oliva, glabras o subglabras en la lámina y en el dorso de la hoja, y pueden crecer entre $15-21 \mathrm{~cm}$ de largo y $5-8 \mathrm{~cm}$ de ancho, con un pecíolo pequeño de $1 \mathrm{~cm}$. Las flores tienen un cáliz persistente y los pétalos que forman la corola son obtusos. El número de estambres es el mismo que para los pétalos, con anteras oblongas. El ovario sésil, subgloso, generalmente de 4 a 6 células (lóculos) su fruto es una baya verde globosa de casi $1 \mathrm{~cm}$ de ancho. Sus componentes principales son la metilxantina, teobromina, teofilina, guanidina y esteroides, además, aceites esenciales, acido nicotínico, ácido ascórbico, riboflavina, piridoxina y triterpenos. A partir del extracto acuoso y etanólico de sus hojas se ha identificado taninos y flavonoides (Sequeda-Castañeda et al. 2016). Las estructuras de sus metabolitos se muestran en la Figura 4. Dentro de sus usos tradicionales, se utiliza las hojas secas para preparar una infusión (5 a $10 \mathrm{~g}$ en un litro de agua), para tratar la diabetes e Intoxicación de la sangre, el número de dosis (tazas al día) dependiendo de la necesidad del paciente (Bussmann \& Douglas 2015). El extracto etanólico y acuoso de sus hojas, se utiliza en la Amazonía para el tratamiento de dolor de riñones, fiebres en malarias, digestivo e hipoglicemiante (Saavedra G. 2017). Presenta efecto antibacteriano frente a Staphylococus aureus (MIC $16 \mathrm{mg} / \mathrm{mL}$ ) y Escherichia coli (MIC $128 \mathrm{mg} / \mathrm{mL}$ ), teniendo como fármacos referentes a la tetraciclina y amoxicilina respectivamente (Bussmann et al. 2010). Es una de las plantas que presenta menor toxicidad, LC $\mathrm{C}_{50}$ $>10000 \mu \mathrm{g} / \mathrm{mL}$ (Bussmann et al. 2011).<smiles>Cn1cnc2c1c(=O)[nH]c(=O)n2C</smiles><smiles>CN1C(=O)C2C(N=CN2C)N(C)C1=O</smiles><smiles>CN1C(=O)C2NC=NC2N(C)C1=O</smiles>

Metilxantina

Teobromina

Teofilina<smiles>Cc1cc2nc3c(=O)[nH]c(=O)nc-3n(C[C@H](O)[C@H](O)[C@H](O)CO)c2cc1C</smiles>

Ácido nicotínico

Ácido ascórbico Riboflavina<smiles>Cc1ncc(CO)c(CO)c1O</smiles>

Piridoxina

Figura 4. Estructuras de metabolitos principales de "agracejo" Ilex guayusa Loes

Vallesia glabra Cav. (Apocynaceae): Posee una amplia distribución en América (Castañeda Montoya 2018) a esta especie se le conoce como agracejo, cun cun o cuncuno. Posee el aspecto de un arbusto, aunque pueden referirse también como árbol, cuya altura está alrededor de 3 a $5 \mathrm{~m}$, no obstante, existen variedades en México que van desde 1,5 a $3 \mathrm{~m}$; forma parte de los algarrobos peruanos y puede 
encontrarse en hábitad desde los 30 a $1300 \mathrm{~m}$ de altura en las regiones Nor-costeras (Mostacero et al, 2002). Dentro de sus características botánicas presenta, hojas lanceoladas a elípticas, glabras por ambos lados, inflorescencia en cimas paniculadas, opositifolias, paucifloras, más cortas que las hojas. Flor pequeña con disminuida presencia de olor, tubular, blanca verdosa, con borde en forma de estrella. Presenta gineceo bicarpelar, ovario bicarpelar, bilocular y cada lóculo contiene 4 óvulos. Sus frutos son drupas colgantes, de color blancas o perlados, traslúcido, oblongas. Semilla: ovoide, marrón claro a blanquecina, longitudinalmente

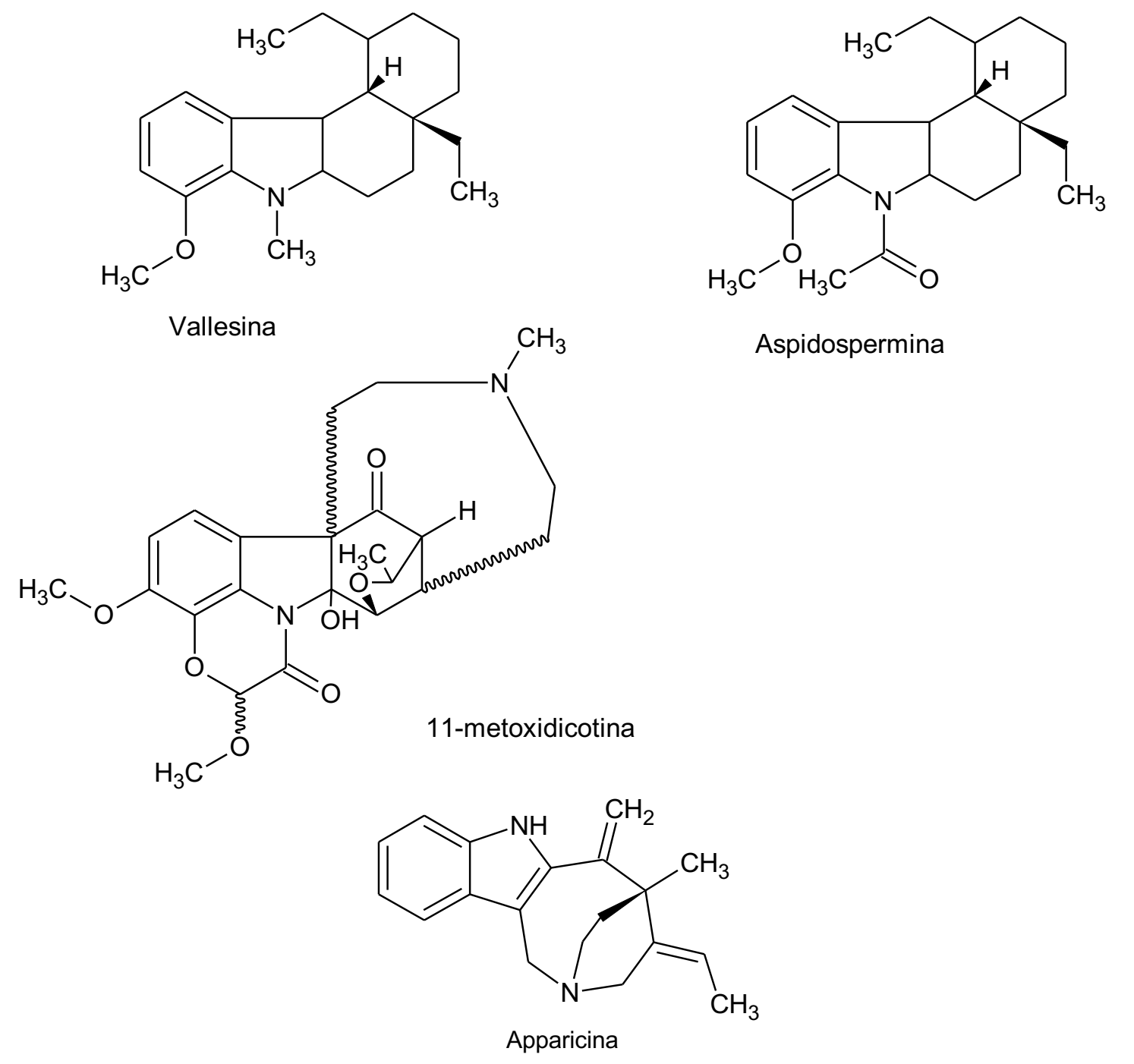

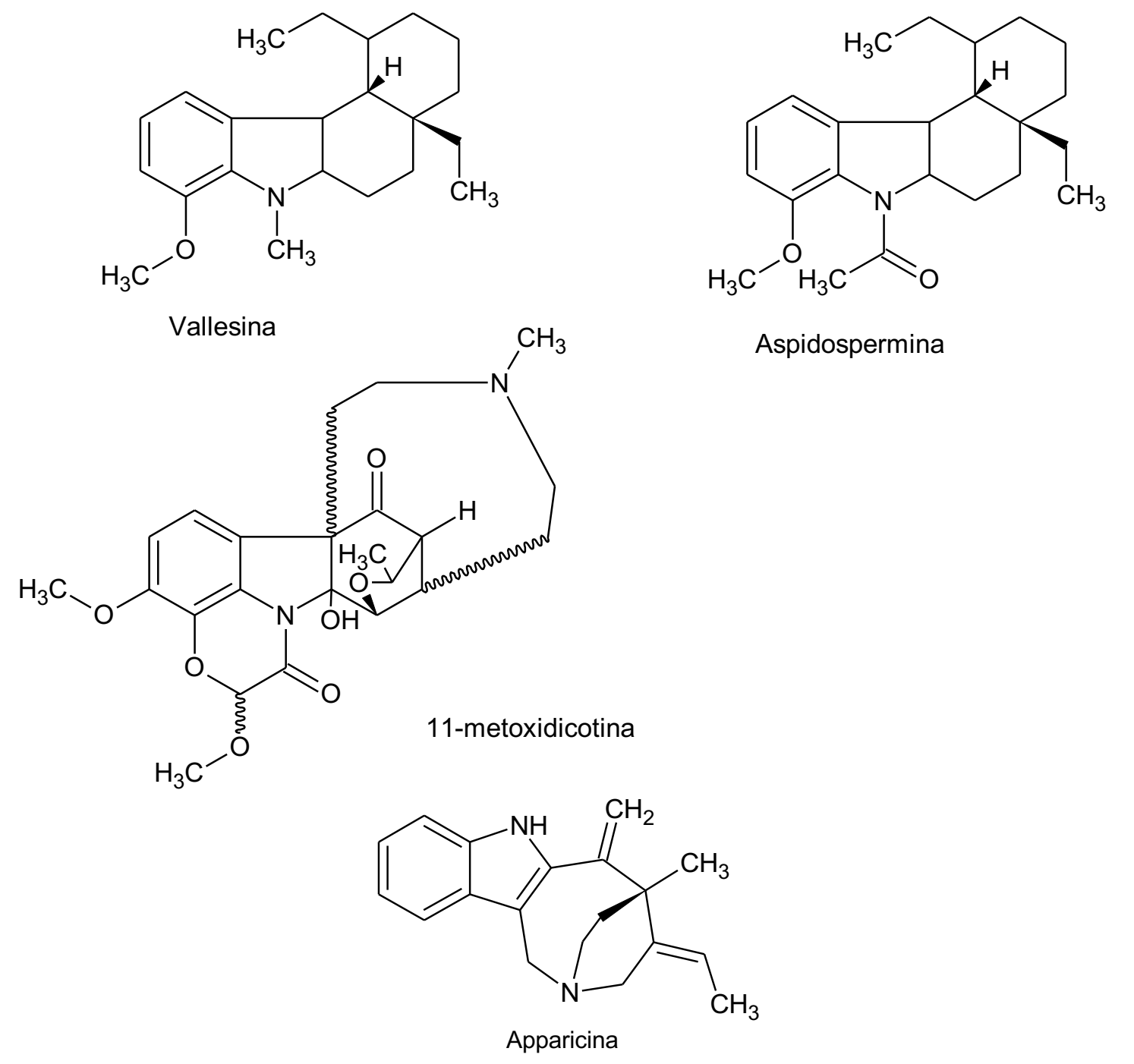

surcada. 6-8 mm de largo por 2,5-3 mm de diámetro (Castañeda Montoya 2018, Guadalupe \& Albornoz 2013).

Presenta alcaloides indólicos tal como la vallesina, aspidospermina, 11-metoxdicotina, apparicina, tubotaïwine, vincadifformina, condilocarpina, (-)razinilam, aspidospermatina, haplocidina y 18oxohaplocidina, se han aislado de las hojas y tallos de una especie de Vallesia glabra (Cav) boliviana (Zèches et al. 1995), sus estructuras se muestran en la Figura 5.

Figura 5. Estructuras de alcaloides más importantes con actividad terapéutica de Valesia glabra

Vallesia glabra, en el Perú tiene gran utilidad y diversas propiedades medicinales, como antiinflamatorio ocular, trastornos ulcerativos gastrointestinales, las hojas frescas preparados en decocto, son utilizadas para el tratamiento de mordeduras de serpientes e hipoglicemiante (Bussmann \& Douglas 2015), además el decocto de tallos para el tratamiento del escozor en alergias y varicela. El extracto etanólico y acuoso de la planta tiene actividad antibacteriana frente a Escherichia coli (MIC $64 \mathrm{mg} / \mathrm{mL}$ y $32 \mathrm{mg} / \mathrm{mL}$ respectivamente) y sólo el extracto etanólico tiene actividad frente a Staphylococus aureus (MIC $16 \mathrm{mg} / \mathrm{mL}$ ) (Bussmann et al. 2010) además, el extracto hidroalcóholico y el 
aceite esencial, de las hojas presentan un ligero efecto inhibitorio de Staphylococus aureus meticilino resistente 12 y $13 \mathrm{~mm}$ de halo de inhibición respectivamente, además también presentan efecto inhibitorio sobre el crecimiento de Pseudomona auriginosa (7 mm) (Mogollón \& Rodríguez. 2019). Por otro lado, las hojas molidas o como extracto de estas se utilizan como antipiréticos, el emplasto del fruto como antimicótico, y la planta triturada como tratamiento para el acné leve a moderado. Finalmente, en la agricultura, se utiliza para la aumentar fertilidad del suelo, como hábitat para animales y alimento para aves (fruto maduro), fungicida, para controlar las enfermedades de la papa (Whaley et al 2010). Es una de las plantas de baja toxicidad, $\mathrm{LC}_{50}>10000 \mu \mathrm{g} / \mathrm{mL}$ (Bussmann et al. 2011).

Perú es el cuarto país con mayor diversidad de plantas, a nivel del continente Americano, pues presenta 19147 especies y de ellos 7590 son restringidos (Ulloa et al 2017), la mayor parte de sus investigaciones etnobotánicas tienen características etnomedicinales (La Torre-Cuadros 2006). La información científica etnobotánica y etnofarmacológica peruana sobre el agracejo como depurador y detoxificante, en sus diferentes especies, es escasa, no obstante, se muestran muchas investigaciones internacional que aportan diversas experiencias farmacológicas (Tabla 4), la mayor cantidad de investigaciones provienen de países como Irán, Pakistan, México, argentina y Bolivia, quienes muestran resultados sobre la actividad fitoquímica y terapéutica. El tener información internacional de una especie en común, por ejemplo, B.vulgaris en Irán y Perú, debería servir de base para evaluar la bioequivalencia de sus metabolitos y sus respectivas actividades etnofarmacológicas, puesto que la concentración de metabolitos se ve influenciado por la altitud sobre el nivel de mar y características geográficas del país y zona cultivada, no obstante, su cuantificación depende de la droga vegetal utilizada y el método de extracción (Yepes \& Silviera. 2011). En la Tabla 5, se presenta de manera cualitativa la presencia de fitoconstituyentes en las 3 especies, la misma que podría ser útil para su caracterización, cuantificación y ser el principal factor que inicie la diferenciación biológica para el reconocimiento de las diversas especies, de no ser así, podría ser una de las razones que conteste las muchas dudas sobre el éxito de la etnomedicina y la aplicación de la fitoterapia e incurrir en la homonimia botánica. Las informaciones que provienen de tesis universitarias de pregrado y postgrado, han aportado con información fitoterapéutica peruana es muy importante, aunque si no se divulga dentro de círculos científicos y no se encuentre publicada, no podrán contribuir a la consolidación y sistematización de la fitofarmacología nacional (Cáceres G. 2014).

\section{Desafíos Actuales y futuros}

Actualmente la información científica peruana del agracejo es escasa, lo que debería generar en las instituciones el punto de inicio para la sistematización de la información etnobotánica en una Farmacopea, esto generará que se aproveche mejor los recursos vegetales, aperturas a nuevas investigaciones y mejoraras de los servicios de atención primaria de salud en el Perú, debido a que reduciría el gasto en compras y consumo de medicamentos convencionales.

\section{Conclusiónes}

La escasa de información científica etnobotánica y etnomedicina peruana, genera un riesgo de adaptación del conocimiento científico internacional a las especies oriundas, esta falta de información, generará homonimia, quien podría ser el factor principal en la confusión de en la recolección de las especies vegetales con características fitológicas similares. Por lo tanto, para que una especie vegetal se distribuya como producto herbario acabado, tiene que tener, además del sustento científico internacional, el nacional y local, por ello, proponemos que toda la información nacional y local aporte a obtener un registro nacional o farmacopea peruana, de lo contrario, se estaría en una situación donde se tiene muchas especies homónimas sin sustento etnobotánico y etnofarmacológico científico, y seguir consumiendo una especie vegetal sin información comprobada que estaría influenciando sobre la efectividad de la indicación terapéutica.

Tabla 4. Número de investigaciones encontradas en base de datos sobre el agracejo: etnobotánica y actividades farmacológicas

\begin{tabular}{cccccc}
\hline & Buscador & $\begin{array}{c}\text { Vallesia glabra } \\
\text { (Cav.) }\end{array}$ & $\begin{array}{c}\text { Ilex } \\
\text { guayusa } \\
\text { Loes }\end{array}$ & $\begin{array}{c}\text { Berberis } \\
\text { vulgaris }\end{array}$ & $\begin{array}{c}\text { berberis } \\
\text { rigida }\end{array}$ \\
\hline Peruanas & Google académico & 6 & 6 & 3 & 0 \\
\hline \multirow{2}{*}{ internacional } & PubMed & 4 & 18 & 836 & 0 \\
\cline { 2 - 6 } & Scopus & 0 & 11 & 527 & 0 \\
\hline Total & & 10 & 35 & 1366 & 0 \\
\hline
\end{tabular}


Tabla 5. Fitoconstituyentes comunes que poseen el extracto acuoso de las hojas, tallos, raíces y frutos del agracejo en las diversas especies.

\begin{tabular}{|l|l|l|l|l|}
\hline METABOLITOS & ENSAYOS & $\begin{array}{l}\text { Berberis } \\
\text { vulgaris L }\end{array}$ & $\begin{array}{l}\text { llex guayusa } \\
\text { Loes }\end{array}$ & $\begin{array}{l}\text { Vellesia glabra Ruiz } \\
\text { \& Pav. }\end{array}$ \\
\hline Compuestos lactónicos & Baljet & - & - & + \\
\hline Esteroides & $\begin{array}{l}\text { Liebermann- } \\
\text { Burchart }\end{array}$ & + & + & + \\
\hline Alcaloides & Dragendorff & + & + & + \\
\hline Alcaloides & Mayer & + & + & + \\
\hline Alcaloides & Wagner & + & + & + \\
\hline Resinas & Resinas & + & - & - \\
\hline Azucares reductores & Fehling & + & + & - \\
\hline Saponinas & Espuma & - & + & + \\
\hline Fenoles & Cloruro férrico & + & + & - \\
\hline Aminoácidos & Ninhidrina & + & - & - \\
\hline Quinonas & Borntrager & - & + & - \\
\hline Flavonoides & Shinoda & - & + & + \\
\hline Antocianinas & Acido-Base & + & - & + \\
\hline Catequinas & Carbonato de sodio & - & - & - \\
\hline Taninos & Gelatina NaCl. & - & + & + \\
\hline
\end{tabular}

$(+)$ : presente, (-): ausente

\section{Declaraciones}

Lista de abreviaturas: MC-medicina complementaria; MA-medicina alternativa; $\mathrm{MN}-$ medicina natural; MT-medicina tradicional; NCCAMNational Center for Complementary and Altermative Medicine; OPS-Organización Panamericana de la Salud; EsSalud-Seguro social de salud del Perú.

Consentimiento para la publicación: No aplica Disponibilidad de datos y materiales: No aplica Conflicto de intereses: Los autores declaran no tener conflicto de interés alguno

Financiamiento:Programa doctoral financiado por FONDECYT-Banco Mundial (Contrato N07-2018FONDECYT/BM-Programas de Doctorados en Áreas estratégicas y Generales "Doctorado en Farmacia y Bioquímica").

Contribuciones de autores: CNRS buscó y analizó la literatura y contribuyó principalmente en la redacción de la revisón, VEVL Buscó y analizó la literatura y adaptó las figuras, SVA analizó la literatura y revisó la ortografía y JKR buscó literatura y configuró la bibliografía.

\section{Agradecimientos}

Con gratitud al Dr. Rainer W. Bussmann, por los conocimientos y aportes al revisar este manuscrito, a cada uno de los colegas del grupo de investigación del doctorado en farmacia y bioquímica que aparecen en esta revisión por su invaluable contribución a la redacción de este artículo.

\section{Literatura citada}

Alva E. 2017. Etnobotánica y características morfológicas de la vegetación leñosa en un remanente de bosque de la microcuenca Río
Grande, la encañada - Cajamarca. Tesis de la Universidad Nacional de Cajamarca. Cajamarca, Perú.

Aredo V, Carranza-Cabrera J, Siche R. 2017. Inventario de especies vegetales de La Libertad (Perú) y análisis de su potencial agroindustrial. Agroindustrial Science 7:87-104.

Bussmann RW, Glenn A, Meyer K, Rothrock A, Townesmith A, Sharon D, Díaz D, Castro $M$, Cardenas R, Regalado S, Del Toro R, Chait G, Malca G, Perez F.2009a. Antibacterial Activity of Medicinal Plants of Northern Peru - Part II. Arnaldoa 16:93103.

Bussmann RW, Glenn A, Meyer K, Rothrock A, Townesmith A, Sharon D, Díaz D, Castro $M$, Cardenas R, Regalado S, Del Toro R, Chait G, Malca G, Perez F.2009b. Phyto-Chemical Analysis of Peruvian Medicinal Plants. Arnaldoa 16:105-110.

Bussmann RW, Malca G, Glenn A, Sharon D, Chait G, Díaz D, Pourtmand K, Jonat B, Somogy S, Guardado G, Aguirre C, Chan R, Meyer K, Kuhlman A, Townesmith A, Effio-Carbajal J, Frías-Fernandez F, Benito M.2010. Minimum inhibitory concentrations of medicinal plants used in Northern Peru as antibacterial remedies. Journal of Ethnopharmacology 132:101-108.

Bussmann RW, Malca G, Glenn A, Sharon D, Nilsen $B$, Parris B, Dubose D, Ruiz D, Saleda J, Martinez M, Carillo L, Walker K, Kuhlman A, Townesmith A.2011. Toxicity of medicinal plants used in traditional medicine in Northern Peru. Journal of Ethnopharmacology 137:121-140. 
Bussmann RW, Sharon D. 2015. Plantas medicinales de los andes y la amazonia-la flora mágica y medicinal del norte del Perú. Trujillo, Perú.

Cáceres G. 2014. La importancia de publicar los resultados de Investigación. Revista Facultad de Ingeniería. 23:7-8.

Castañeda Montoya, Nathalie. 2018. Vallesia glabra (Cav.) Link. Lima-Perú.1-6

Carpio J. 2017 Flora arbórea y arbustiva del bosque de Ustuna, centro poblado Santa Isabel de Chumbes, distrito Ocros, provincia Huamanga. Ayacucho, 2016. Tesis de la Universidad Nacional De San Cristóbal De Huamanga. Ayacucho, Perú.

Dueñas JF, Christopher Jarrett, Cummins I, LoganHines E. 2016. Amazonian Guayusa (Ilex guayusa Loes.): A historical and ethnobotanical overview. Economic Botany 70:85-91.

EsSalud. 2016. Vida y salud integral. Boletín Informativo de Medicina Complementaria. Vol.8. http://www.essalud.gob.pe/downloads/boletin-mecseptiembre-2016.pdf.

EsSalud. 2008. Petitorio nacional de productos, recursos e insumos terapéuticos afines de uso en medicina complementaria. Lima-Perú.

Galán de Mera A, Sánchez I, Montoya J, Linares E, Campos J, Vicente J. 2017. La vegetación del norte del Perú: de los bosques a la Jalca en Cajamarca. Acta Botanica Malacitana 40:157.

Guadalupe G, Albornoz P. 2013. Anatomía Foliar De Vallesia Glabra (Apycynaceae), Especie De Importancia Medicinal Y En Frugivoría. Lilloa 50:2532.

Gutiérrez Y, Linares L. 2012. Etnobotánica y fitoquímica de plantas tintoreas En Las Comunidades De Rumira, Chaullacocha Y Chupani: Provincia De Urubamba- Cusco. Tesis de la Universidad Nacional de San Antonio Abad del Cusco. Cusco, Perú.

Hanachi P, Kua SH, Asmah R, Motalleb G, Fauziah O. 2006. Cytotoxic Effect of Berberis vulgaris fruit extract on the proliferation of human liver cancer line (HepG2) and its Antioxidant Properties. International Journal of Cancer Research 2:1-9.

Javadzadeh S, Reza S. 2012. Therapeutic application of different parts Berberis Vulgaris. International Journal of Agriculture and Crop Sciences 4:404-408.

Javadzadeh S, Ebrahimi A. 2013. The traditional uses and pharmacological effects of different parts Berberis vulgaris (Berberine) in Iran. Scientia 1:6166.
Kalmarzi, RN, Naleini SN, Ashtary-Larky D, Peluso I, Jouybari L, Rafi A, Ghorat F.2019. Anti-inflammatory and immunomodulatory effects of Barberry (Berberis Vulgaris) and its main compounds. Oxidative medicine and cellular longevity. doi:10.1155/2019/6183965.

Khan I, Najeebullah S, Muhammad Ali M, Khan Z. 2016. Phytopharmacological and ethnomedicinal uses of the Genus Berberis (Berberidaceae): A review. Tropical Journal of Pharmaceutical Research September.15:2047-2057.

Nasiri R, Nima S, Ashtary-Larky D, Peluso LI, Jouybari L, Rafi A, Ghorat F, Heidari N, Sharifian F, Mardaneh J,Aiello P, Helbi S, Kooti W. 2019. AntiInflammatory and Immunomodulatory Effects of Barberry (Berberis vulgaris) and Its Main Compounds. Oxidative Medicine and Cellular Longevity. 1-10

La Torre-Cuadros MA, Alban J. 2006. Etnobotánica en los andes del Perú. En: Moraes-R M, Øllgaard B, Kvist LP, Borchsenius F, Balslev H. (eds) Botánica Económica de Los Andes Centrales, 2006: 239-245.

Minaiyan M, Ghannadi A, Mahzouni P, Jaffari-Shirazi E. 2011. Comparative Study of Berberis vulgaris Fruit Extract and Berberine Chloride Effects on Acetic Acid-Induced Colitis in Rats. Iran J Pharm Res. 10:97-104.

Mogollón M, Rodríguez I. 2019. Efecto del extracto hidroalcohólico y aceite esencial

de hojas de Vallesia glabra sobre el crecimiento de Staphylococcus aureus meticilino resistente y Pseudomonas aeruginosa. PuebloCont. 30: 299306.

Llanos J. 2012. Etnobotánica de la flora arbórea y arbustiva del Departamento de Cajamarca. Tesis de la Universidad Nacional de Cajamarca. Cajamarca, Perú.

Mahmoudvand $H$, Ayatollahi $S$, Sepahvand A, Sharififar F, Ezatpour B, Gorohi F, Saedi E, Jahanbakhsh S. 2014. Antifungal, Antileishmanial, and Cytotoxicity Activities of Various Extracts of Berberis Vulgaris (Berberidaceae) and Its Active Principle Berberine. ISRN Otolaryngology, January, 1-6.

Marinoff MA, Martínez JL, Urbina MA. 2009. Precauciones en el empleo de plantas medicinales. Boletín Latinoamericano y del Caribe de Plantas Medicinales y Aromáticas 8184-187.

Minga D, Ansaloni R, Verdugo A, Ulloa C. 2016. Flora Del Páramo Del Cajas. Ecuador. Universidad del Azuay. Ecuador Cuenca.

Mokhber-Dezfuli N, Saeidnia S, Gohari A, Kurepaz- 
Mahmoodabadi M. 2014. Phytochemistry and pharmacology of berberis species. Pharmacognosy reviews. 8. 8-15.

Mostacero-León J, Mejía-Coico F, Gamarra-Torres O.2002.Taxonomía de las fanerógamas útiles del Perú. Trujillo, Perú. Perú.

Instituto Nacional del cáncer. 2015. Medicina complementaria y alternativa-Salud Juntos https://www.cancer.gov/espanol/cancer/tratamiento/ mca. (Consultado 24-01-2020)

Organización Mundial de la Salud. Medicina tradicional: definiciones. https://www.who.int/topics/traditional_medicine/defi nitions/es/_(consultado 23/01/2020)

Organización Panamericana de la Salud. Situación de las plantas medicinales en Perú 2018. www.paho.org. (Consultado 24-01-2020)

Radice M, Vidari G. 2007. Caracterización fitoquímica de la especie llex guayusa Loes y elaboración de un prototipo de fitofármaco de interés comercial. La Granja. Revista de Ciencias de la Vida 6:3-11.

Rahimi-Madiseh M, Lorigoini Z, ZamaniGharaghoshi H, Rafieian-Kopaei M. 2017. Berberis Vulgaris: Specifications and Traditional Uses. Iranian Journal of Basic Medical Sciences 20: 569-87. doi:org/10.22038/IJBMS.2017.8690.

Rodriguez E, Almedia S. 2009. Evaluación del potencial genotóxico de cinco especies medicinales de uso popular en el Perú. Tesis de la Universidad Nacional de La Amazonía Peruana. Iquitos, Perú

Roersch C.1994. Plantas Medicinales en el sur Andino del Perú. Centro de Medicina Andina. CuscoPerú; Pp: 907-910

Saavedra S. 2017. Caracterización de los arbustos en la provincia de Tarma, departamento de Junín, con énfasis en su morfología vegetativa. Tesis de la Universidad Nacional Agraria la Molina. Lima, Perú.

Saavedra G. 2017. Determinación de la concentración de Cafeína en Guayusa (Ilex Guayusa Loes), En San Ignacio-Cajamarca. Tesis de la Universidad Nacional de Cajamarca. Jaén, Perú.10

Salehi B, Selamoglu Z, Sener B, Kilic M, Kumar A, de Tommasi N, Sinisgalli C, Milella L, Rajkovic J, Morais-Braga M, Bezerra C, Rocha J, Coutinho H, Oluwaseun A, Khan Z, Ahmad S, Erol E, Ali Z, Ostrander E, Sharifi-Rad J, Cádiz-Gurrea M, Taheri Y, Martorell M, Segura-Carretero A, Cho W. 2019. Berberis Plants-Drifting from Farm to Food Applications, Phytotherapy, and Phytopharmacology. Foods. 8:522
Sequeda-Castañeda L, Modesti Costa C, Gamboa F, Gutiérrez S, Luengas P. 2016. Ilex guayusa Loes (Aquifoliaceae): Amazon and Andean native plant. Pharmacologyonline 3: 193-202

Srivastava S, Srivastava M, Misra A, Pandey G, Rawat A. 2015. A review on biological and chemical diversity in Berberis (Berberidaceae). EXCLI journal.14.247-267.

Tropicos Org. Berberis Vulagaris L. 2020. www.tropicos.org/name/3500015. (Consultado 2401-2020)

Ulloa C, Acevedo-Rodríguez P, Beck S, Belgrano M, Bernal R, Berry P, Brako L. 2017. An integrated assessment of the vascular plant species of the Americas. Science 358 (6370): 1614-17.

Ulloa C, Sagástegui A, Sánchez I. 2006. Berberidaceae endémicas del Perú. Revista Peruana de Biología 13: 879-891.

Vásquez R, Rojas R. 2016. Clave para identificar grupos de familias de Gymnospermae y Angiospermae del Perú. Lima, Perú. Jardín Botánico de Missouri

Whaley O, Orellana A, Pérez E, Tenorio M, Quinteros F, Mendoza M. 2010 Plantas y vegetación de Ica, Perú. Royal Botanic Gardens Kew. Lima, Perú.

Yepes A, Silveira M. 2011.Respuestas De Las Plantas Ante Los Factores Ambientales Del Cambio Climático Global (Revisión). Colombia Forestal 14:213-232.

Zèches, M, Mesbah K, Richard B, Moretti C, Nuzillard JM, Men-Olivier L. 1995. Alkaloids from leaves and stems of Vallesia glabra. Planta Medica 61:89-91. 